\title{
Proposed Design of a Real-Time COVID-19 Pandemic Contact Tracing Using Mobile Phone
}

\section{ESJ Natural/Life/Medical Sciences}

\section{Ezenwa Nwawudu,}

Global Needs Institute,Saint Etienne,France

\section{Amanze Ikwu, \\ Cardiology Department \\ University Hospitals Plymouth NHS \\ Trust,Plymouth,United Kingdom}

\section{Ugochi Ikwu,}

Senior Enterprise Engineer, Allergan USA.

New Jersey,USA

\section{Nneoma Oparah,}

Argon Medical Devices,

Plano,Texas,USA

\section{Stanley Nnorom,}

Educational Management and Policy

Faculty of Education

Nnamdi Azikiwe University, Awka,Nigeria

Submitted: 24 August 2020

Accepted: 04 December 2020

Published: 31 December 2020

Corresponding author:

Dr. Amanze N.Ikwu

DOI: 10.19044/esj.2020.v16n36p1

c) Copyright $2020 \mathrm{Ikwu} \mathrm{A}$,

Distributed under Creative Commons

BY-NC-ND 4.0 OPEN ACCES

\section{Cite as:}

Nwawudu.E, Ikwu.A.,Ikwu.U., Oparah.N., Nnorom.S. (2020). Proposed Design of a Real-Time COVID-19 Pandemic Contact Tracing Using Mobile Phone. European Scientific Journal, ESJ, 16 (36), 1. https://doi.org/10.19044/esj.2020.v16n36p1

\begin{abstract}
As the virus that causes COVID-19 continues to spread from person to persons in communities and rampaging the world, the need for an effective real-time surveillance system becomes paramount. Advance contact tracing and detection of the persons with the virus represents one of the main strategies to prevent transmission. Although COVID-19 surveillance systems such as contact tracing mobile apps have improved the administration and management of virus, there are still challenges such as privacy, cost and ethical issues, the adoption of new technologies, standardized cases, and validly diagnosed case and validity. However, the current mobile apps contact tracing system adopted by different nations has complemented conventional tracing effort in fighting the virus. This proposal is a model for an interactive computer system using mobile phones and the internet for real-time collection and transmission of events related to COVID19. It will aid the administration and presumptive management of COVID-19 in the world, especially in rural areas. This proposal shows that a sophisticated COVID-19 surveillance system can be build using mobile phones with the right telecommunication technology partner.
\end{abstract}

Subject: Public Health

Keywords: COVID-19, Contact tracing, Mobile phone, Real-time, Pandemic 


\section{Introduction}

With the continued spread of the COVID-19 pandemic; and the vaccine for it still on trial. The world is pinning its hopes on fighting the spread of the ability to quickly identify people who might have been exposed to the virus [1]. Contact tracing is a key public health intervention and a critical component of comprehensive strategies to control the spread of COVID-19. Breaking the chains of human-to-human transmission through contact tracing by identifying people exposed to confirmed cases, quarantining them, monitoring them to ensure rapid isolation, and testing and treatment in case they develop symptoms is very essential in checkmating the spread of the COVID-19 pandemic. When implemented systematically and effectively, these actions can ensure that the number of new cases generated by each confirmed case is contained below one [2]. Many researchers and developers are working closely with public health authorities in exploring different options to support contact tracing with technological tools. Since the first use of mobile technology app to support contact tracing in Singapore, many EU/EEA countries and other developed countries have designed a mobile app to complement the conventional contact tracing efforts [3]. However, the majority of countries in the world still rely on manual contact tracing alone which is generally a laborious, slow process that relies on in-person interviews and detective work.

Contact tracing in the context of COVID-19 requires identifying persons who may have been exposed to a person with COVID-19 and monitoring them up daily for 14 days from the last point of exposure. Contacts should remain in self-quarantine during the 14-day monitoring period in order to limit the possibility of exposing other people to infection should they become ill, since COVID-19 transmission can occur before symptoms develop. When implementing contact tracing, the following should be put into consideration: community engagement and public support; proper planning and consideration of local contexts, communities, and cultures; a workforce of trained contact tracers and supervisors; logistics support to contact tracing teams; and well-designed information systems to collect, manage, and analyse data in real-time [4].

Contact tracing has challenges such as incomplete identification of contacts, inefficiencies in paper-based reporting systems, complex data management requirements, and delays in steps from the identification of contacts to the isolation of suspected cases among contacts. Technological tools can play a role in overcoming some of these challenges when integrated into a sufficiently resourced contact tracing program. Digital tools work effectively for contact tracing when integrated with an existing public health system that includes health services personnel, testing services, and manual contact tracing infrastructure. [5] 


\section{Review of the literature}

The applications involving the use of mobile phones and the internet have been of interest to many researchers recently. For example, Voxiva developed a system called Alerta that the phone and internet for communication and disease surveillance. Rural health workers can report new cases of disease systematically and in real-time by calling into Voxiva's system and pushing buttons on the phone. Alerta project was piloted initially by the health clinic of the Ministry in Peru.[6] It was later in October 2002 implemented in the Peruvian Navy. Alerta led to an early outbreak identification response, timely case management, and increase reviews of clinical procedures within reporting units. They concluded that Alerta represented a fully functional alternative for cost-effective real-time disease surveillance [7].

Similar systems were developed and deployed in the developing world: Latin America, Iraq, India, and Africa using the Voxiva platform for health solutions. For example, in Africa, Voxiva created a national HIV/AIDS information system for eight countries that monitors current data for national and global reporting requirements to reduce the spread of viral resistance. In India, within a month, a surveillance system for Japanese encephalitis was created by Voxiva [6].

The project Cell Life is using cell phones to monitor adherence for the management of HIV/AIDS in patients on antiretroviral therapy in South Africa [8]. Most of the platforms Cell Life project uses include a global system for mobile communications (GSM), wireless Internet gateway (WIG), and a geographical information system (GIS) database.

Research reports show that public health informatics and disaster response system can be build using Java-enabled wireless phones [9]. The research stressed that Java-enabled wireless phone technology was inherently deployable and portable. The minimal orientation for new hardware is needed since everyone entered the phone keypad and pushing the button.

Also, The Gota software application,[10] created by WHO with partners of the Global Outbreak Alert and Response Network, significantly increased the contact tracing performance of the Ebola virus in 2019 in the Democratic Republic Congo.[11].

\section{Objectives}

Our overall goal in this study is to develop a robust interactive COVID-19 pandemic contact tracing system using mobile phones and the internet that will aid real-time collection and transmission of adverse events related with administration, surveillance and management of COVID-19 pandemic in the world, especially in rural areas. 


\section{Method}

\subsection{The System Design}

This proposed system can aid in outbreak response and symptom tracking aspects of contact tracing include case investigation, listing and monitoring of contacts, and automating analysis and performance monitoring. This proposed system will manage dynamic relationships between cases and contacts because contacts may have multiple links.

This proposed system will also optimize the workflow of field workers conducting contact tracing as well as providing functionality for supervisors to monitor the implementation of contact tracing. This system will combine the phone and the Internet to create a real-time contact tracing system of COVID-19 pandemic as a complement of the manual contact tracing. It will be an interactive voice response application for mobile phones in English and French, based on a system from a chosen telecommunications company based in the country.

This proposed system will have five elements in the architectural design. The five architectural elements are: the central database and Web server, remote access to the database from any Internet-connected computer, telephone audio computer-assisted personal interviewing, voice messages, and Short Message Service (SMS)-based communications to and from the server via the mobile phones (Figure 1).

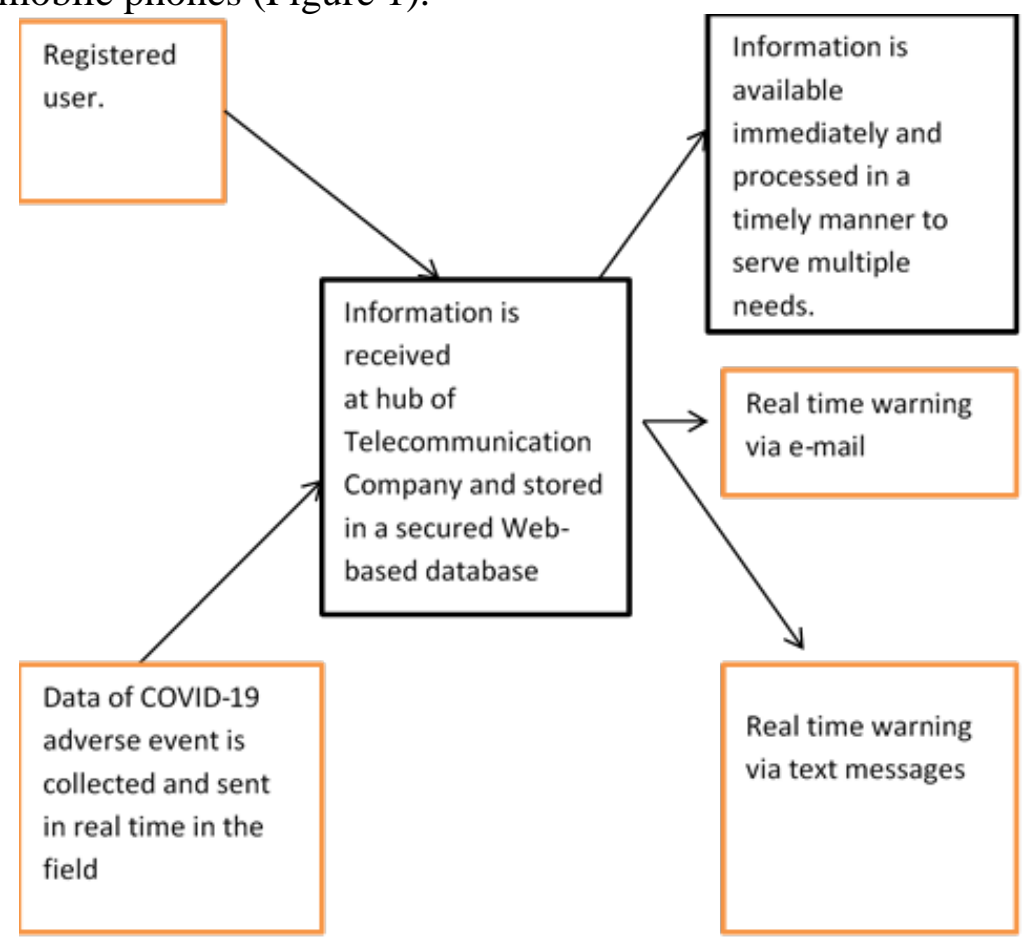

Figure 1: Diagram of the System Architecture 


\subsection{How the system will work}

You can register on the system using a mobile phone and follow the instructions on a simple prompted menu, and enter your name, age range, mobile phone number, and postcode. A confirmation text message will be sent to you to say that you have successfully registered in the system.

When you have symptoms of COVID-19, you log in to your account in the system and make your report, either by filling a short questionnaire or voice note.

The designated health officials will receive the information immediately via the internet, analyse the data, and use the system's communication and messaging tools to respond. The designate health officials can monitor incoming reports through a Web interface. Individual event reports arrived in real-time with full-case details.

Once the designated health officials analyse the data with COVID-19 systems, they will notify you of the necessary things to do, such as 14 days of selfisolation, wearing a surgical mask, distancing, and test via e-mail and SMS messages. The designated health officials could communicate with remote health officials in that locality to intensify the awareness of COVID-19 guidelines such as the washing of hands, avoid handshaking, social distancing, etc.

If you test positive for COVID-19, the designated health official will ask you to consent to your handshake information via a digital form. A child's parent, guardian, or care will be asked for consent if tested positive.

The filled information enables designated health officials to call close contacts to advise them on what to do. Health officials will not disclose the name of the infected person. Also, the database will only available to authorised health officials.This system will be operational 24 hours a day, seven days a week.

\section{Discussion}

\subsection{Advantages}

Although contact tracing is successfully done manually without such a proposed system, the use of this system will facilitate contact tracing implementation on a large scale. This proposed system will offer an opportunity that will strengthen contact tracing capacity for COVID-19. An example is a significant increase in contact tracing performance of the Ebola virus in 2019 in the Democratic Republic Congo facilitated by the implementation of Go.Data [11].

This system will have advantages such as; includes improved data quality, the ability to provide analysis and real-time situation awareness, being able to trace larger numbers of contacts in a shorter period, and the ability to perform coordination and management of contact tracing teams. Moreover, this system 
will provide information(s) for monitoring and evaluation of the contact tracing.

This system can also be used by government agencies in the field to set up post-COVID-19 health facilities which offer psychological helplines and provision of palliatives to remote areas as well as garner feedback from people [12].

\subsection{Challenges}

There are societal fears of privacy breaches, provision of wrong medical advice based on self-reported symptoms, and the systematic exclusion of some members of society who cannot access the system. So, the need to have sufficient regulatory oversight and ethical issues surrounding privacy, security, transparency, and accountability [13].

As well, there is the possibility that marginalized and disadvantaged groups particularly those in the low ad middle-income will be more likely to be excluded. Again, the use of the system will incur costs such as hardware, and software costs, training costs, and require continuous user support.

Moreover, there is currently limited evidence to evaluate the effectiveness of digital tools for COVID-19 response like such proposed a system, despite several countries and areas have deployed. So should not be considered as 'stand-alone solutions' for contact tracing but rather as complementary tools for manual contact tracing.

\section{Conclusion}

This proposed system will strengthen contact tracing for COVID-19 when implemented. It reveals that COVID-19 contact tracing can be build using mobile phones as feasible means of collecting and reporting data in real-time. The proposal shows that it's not necessary to have the latest laptops or desktop computers to create a sophisticated public health surveillance system. With the partnership of the right telecommunication company partner, a COVID-19 contact tracing system can be build using mobile phones.

Conflict of Interest: The authors declare no conflict of interest

\section{Funding: None}

\section{References:}

1. COVIDSafe app | Australian Government Department of Health. https://www.health.gov.au/resources/apps-and-tools/covidsafe-app

2. Mobile applications in support of contact tracing for COVID-19 A guidance for EU/EEA Member States 10 June 2020 https://www.ecdc.europa.eu/sites/default/files/documents/covid-19mobile-applications-contact-tracing.pdf 
3. World Health Organisation: Digital tools for COVID-19 contact tracing Annex: Contact tracing in the context of COVID-19 2 June 2020 WHO-2019-nCoV-Contact_Tracing-Tools_Annex-2020.1eng.pdf

4. World Health Organization. Contact tracing in the context of COVID19. (Interim Guidance) ( 10 May 2020, https://www.who.int/publicationsdetail/contact-tracing-in-thecontext-of-covid-19)

5. American Civil Liberties Union. Principles for technology-assisted contact-tracing. ACLU White Paper; 2020, (https://www.aclu.org/report/acluwhite-paper-principles-technologyassistedcontact-tracing, 16 April 2020).

6. Prahalad C. The Voxiva Story. The Fortune at the Bottom of the Pyramid: Eradicating Poverty Through Profits First printing ed. New Jersey: Wharton School Publishing; 2005:361-379.

7. Lescano A, Ortiz M, Elgegren R, et al. Alerta DISAMAR: Innovative disease surveillance in Peru. Paper presented at: Annual Meeting of the American Society of Tropical Medicine and Hygiene Philadelphia, 2003.

8. Cell-Life. The Cell-Life Solution. Available at: http://www.celllife.org/ Accessed: March 1, 2005.

9. Karras BT, Huq SH, Bliss D, Lober WB. National Pharmaceutical Stockpile drill analysis using XML data collection on wireless Java phones. Proc AMIA Symp. 2002:365-391. [PMC free article] [PubMed] [Google Scholar]

10. World Health Organization. Go.Data (Website) https://www.who.int/godata

11. World Health Organization. Speeding up detection to slow down Ebola: smartphone app is game-changer for contact tracing in hotspots in the Democratic Republic of the Congo (News) (https://www.afro.who.int/news/speeding-detectionslow-down-ebolasmartphone-app-game-changercontact-tracing-hotspots, September 2019).

12. Ikwu AN, Igwe DC, Nwawudu SE, Adebayo AS. The Impact of COVID-19 Pandemic on Africa's Healthcare System and Psychosocial Life. Eur J Med \& Nat studies, 2020; 4(2): 57-66

13. http://journals.euser.org/index.php/ejmn/article/view/4797

14. World Health Organization. Ethical considerations to guide the use of digital proximity technologies for COVID-19 contact tracing (Interim Guidance) (https://www.who.int/publications-detail/WHO2019nCoV-Ethics_Contact_tracing_apps-2020.1 , 28 May 2020) 5. World Health Org 